\title{
A Randomized Control Trial of Patient-Controlled Intravenous Analgesia (PCIA) with and Without a Background Infusion Using Sufentanil for Cesarean Section Analgesia
}

\section{Zhong-Biao Nie}

Shanxi Bethune Hospital

Xian-Mei Cui

Shanxi Bethune Hospital

Ran Zhang

Affiliated Hospital of Shanxi University of Traditional Chinese Medicine

Bin Lu

Shanxi Bethune Hospital

Su-Xian Li

Shanxi Bethune Hospital

Tao Cao

Shanxi Bethune Hospital

Ping Zhuang ( $\sim 13700503507 @ 163 . c o m)$

Shanxi Bethune Hospital

\section{Research Article}

Keywords: pain scores, sedation score, postpartum hemorrhage, Injection/attempt (I/A) ratio, satisfaction.

Posted Date: June 2nd, 2021

DOI: https://doi.org/10.21203/rs.3.rs-455428/v1

License: (c) (i) This work is licensed under a Creative Commons Attribution 4.0 International License. Read Full License 


\section{Abstract}

Purpose: Patient-controlled intravenous analgesia (PCIA) is an option for pain cesarean section analgesia. However, the effect of a background infusion on the analgesic requirements of parturients is still debated. In order to attempt to identify the optimal cesarean section analgesia infusion regime, we evaluated the benefit of the background infusion in this randomized study.

Patients and methods: 60 Parturients had a PCIA analgesia protocol initiated consisting of a $0.5 \mathrm{~mL}$ bolus, and compared 2 Groups: no background infusion, 6 min lockout time (Group A). 2 mL/h infusion, 10 min lockout time (Group B). The total amount of sufentanil consumed, two categories of pain scores, sedation score, postpartum hemorrhage, Injection/attempt (I/A) ratio, side effects, satisfaction were calculated for both groups.

Results: Group B significantly reduce visual analog scale®VAS®pain scores 6, 12h. Group A and Group B were not significantly different regarding wound pain at rest (VAS-R) and uterine cramping pain (VAS-U) at postoperative 24, 36 hours. In terms of I/A, Group A was lower than Group B, there was no significant difference. Group B showed significantly higher satisfaction than Group A. Total consumption of sufentanil at 36 hours postoperative was much greater in Group B. Group B can significantly reduce the postpartum hemorrhage within 1 hour. The minimal respiration rates were not significantly different among groups. The side effect were not observed among groups. All parturients had the same Ramsey sedation score.

Conclusion: In comparison with the without background infusion, total consumption of sufentanil at 36 hours postoperative was much greater in the background infusion, but it can significantly reduce the uterine contraction pain and wound pain of 12 hours after cesarean section, significantly improve patient satisfaction, reduce the postpartum hemorrhage within 1 hour, and does not increase the incidence of PONV and respiratory depression in parturients.

\section{Introduction}

It is well known that the pain after cesarean section is usually ranked moderate to severe [1]. After-birth pain management is of vital significance as it can affect whether women can come back to their originally normal social life, and bad management can shorten lactogenesis, thus affecting their caring for their babies [2]. Traditionally, intravenous administration of opioids has been successfully used for pain control after cesarean section [3]. Patient-controlled intravenous analgesia pump (PCIA) is a commonly used method to relieve postoperative pain in hospitalized patients. The method is to titrate analgesics according to demand. Compared with "on-demand" opioid injections, it usually provides better pain control and improved patient satisfaction.

The PCIA prescription involves parameters programmed into the PCIA machine, like background infusion, dose limits, bolus dose as well as lockout interval, with each affecting PCIA efficacy and safety somewhat. Although the panel of American pain society recommends against routine background 
infusion of opioids with PCIA in opioid-naive adults (they are considered that background infusion of opioids can enhance nausea and vomiting, and can cause respiratory depression increase for adults in some research) [4-8], Some of the more studies, have produced contradictory results. Study shows that if a standard morphine PCIA is added with background morphine infusion, the postoperative analgesia can be improved and no side effects will be increased [9]. We show that most of the opioids studied so far are morphine-based. Sufentanil is a type of opioids of which the analgesic potency is strong, and it has enjoyed a wide application for surgery patients during anesthesia, as well as for controlling postoperative pain and relieving labor pain. Grass, J.A 's study shows that a small background infusion may be necessary to sustain analgesia with sufentanil [10].

In order to attempt to identify the optimal cesarean section analgesia infusion regime, we evaluated the benefit of the background infusion in this randomized study.

\section{Results}

From June 2020 to February 2021 a total of 76 parturients were assessed for enrollment the study. 64 parturients were randomly assigned. In the group A, 4 parturients were excluded from this study after randomization, 1 parturients had blood reflux, and 3 parturients adjusted their parameters due to poor analgesia. A total of 60 parturients finally completed this study and were included in the statistical analysis, including 30 cases in the group A and 30 cases in the study group B (see Fig. 1).

The two groups did not show any difference in patient characteristics age, height weight, BMI, Operation time, Gestation, and in-hospital days (Table 1). The $\mathrm{SpO}_{2}$, Blood pressure, respiratory rate, and heart rate values were not significantly different between groups, and hemodynamic data did not show any remarkable changes or difference. Values remained in a normal range during the whole study period.

Table 1 Demographic and obstetric data

\begin{tabular}{|c|c|c|c|}
\hline & Group A $(n=30)$ & Group B $(n=30)$ & $P$ value \\
\hline Age囚year》 & $27.77 \pm 2.775$ & $28.20 \pm 2.87$ & 0.55 \\
\hline Height (cm) & $162.73 \pm 4.456$ & $160.3 \pm 5.032$ & 0.052 \\
\hline Weight (kg) & $72.55 \pm 11.18$ & $71.88 \pm 9.16$ & 0.8 \\
\hline BMI (kg/m2) & $27.34 \pm 3.69$ & $28.00 \pm 3.59$ & 0.489 \\
\hline Operation time $(\mathrm{min})$ & $55.57 \pm 6.92$ & $53.9 \pm 9.89$ & 0.325 \\
\hline Gestation (weeks) & $40 \pm 1$ & $40 \pm 1$ & 0.453 \\
\hline in-hospital days & $4.47 \pm 0.776$ & $4.6 \pm 1.003$ & 0.567 \\
\hline ASA classification, I(II) & $8 \llbracket 22 \rrbracket$ & $6 \rrbracket 24 \rrbracket$ & 0.811 \\
\hline
\end{tabular}


Notes: Values are in the term of mean (Mean $\pm S D)$. BMI = body mass index.

Comparisons of VAS at VAS-R and VAS-U are presented in Table 2. With respect to VAS-R and VAS-U, Group B significantly reduce VAS pain scores $6,12 \mathrm{~h},(P<0.005)$. Group A and Group B did not present any significant difference regarding VAS-R and VAS-U at postoperative 24,36 hours.

Table 2 VAS pain scores 6/12/24/36 hours after surgery for Group A and Group B

\begin{tabular}{|lllll|}
\hline VAS-R & Group A $(\mathrm{n}=30)$ & Group $B(\mathrm{n}=30)$ & $\mathrm{Cl} 95 \%$ & P value \\
\hline $6 \mathrm{~h}$ & $5.37 \pm 1.884$ & $3.43 \pm 1.223^{*}$ & $1.11-2.75$ & $<0.001$ \\
\hline $12 \mathrm{~h}$ & $4.07 \pm 2.180$ & $2.37 \pm 1.402^{*}$ & $0.75-2.64$ & $<0.001$ \\
\hline $24 \mathrm{~h}$ & $2.67 \pm 1.561$ & $2.10 \pm 1.155$ & $-0.14-1.27$ & 0.16 \\
\hline $36 \mathrm{~h}$ & $2.10 \pm 1.213$ & $1.77 \pm 1.073$ & $-0.25-0.92$ & 0.26 \\
\hline VAS-U & Group A $(\mathrm{n}=30)$ & Group B $(\mathrm{n}=30)$ & $\mathrm{Cl} 95 \%$ & P value \\
\hline $6 \mathrm{~h}$ & $5.13 \pm 2.11$ & $3.73 \pm 1.5^{\star}$ & $0.45-2.34$ & $<0.001$ \\
\hline $12 \mathrm{~h}$ & $3.93 \pm 2.32$ & $2.46 \pm 1.47^{*}$ & $0.50-0.46$ & 0.005 \\
\hline $24 \mathrm{~h}$ & $2.63 \pm 1.6$ & $2.1 \pm 1.15$ & $-0.19-1.25$ & 0.15 \\
\hline $36 \mathrm{~h}$ & $1.93 \pm 1.28$ & $1.75 \pm 1.09$ & $-0.49-0.80$ & 0.58 \\
\hline
\end{tabular}

Notes: Values are in the term of mean (Mean $\pm S D$ ). $C l$, confidence interval; ${ }^{*} p<0.05$ relative to Group A.

The number of injections and the number of attempts were significantly lower in Group B at 36 hours, $(P<0.001)$. Group A exhibited lower I/A relative to Group B, there was no significant. Group B showed significantly higher satisfaction than Group A. Total consumption of sufentanil at 36 hours postoperative was much greater in Group $B(P<0.001)$. (Table 3$)$.

Table 3 Attempt, injection, total sufentanil required, I/A, and satisfaction 6/12/24/36 hours after surgery for Group A and Group B 


\begin{tabular}{|c|c|c|c|c|}
\hline & $\begin{array}{l}\text { Group A ( } \\
=30)\end{array}$ & $\begin{array}{l}\text { Group B (n } \\
=30)\end{array}$ & $\mathrm{Cl} 95 \%$ & $\begin{array}{l}P \\
\text { value }\end{array}$ \\
\hline attempt & $84.6 \pm 51.2$ & $7.67 \pm 7.96$ * & $\begin{array}{l}(57.61 \\
96.24)\end{array}$ & $<_{0.001}^{<}$ \\
\hline injection & $69.6 \pm 45.6$ & $5.9 \pm 6.45$ * & $\begin{array}{l}₫ 46.54, \\
80.85 \rrbracket\end{array}$ & $\begin{array}{l}< \\
0.001\end{array}$ \\
\hline I/A & $0.84 \pm 0.16$ & $0.86 \pm 0.24$ & $(-0.13,0.08)$ & 0.60 \\
\hline satisfaction & $3.96 \pm 0.88$ & $4.73 \pm 0.52^{\star}$ & $(-1.14,-0.38)$ & $\begin{array}{l}< \\
0.001\end{array}$ \\
\hline $\begin{array}{l}\text { Total sufentanil consumption at } 36 \mathrm{~h} \\
(\mu \mathrm{g})\end{array}$ & $51.9 \pm 40.1$ & $109.2 \pm 17.9 *$ & $\begin{array}{l}(-73.32, \\
-41.19)\end{array}$ & $\stackrel{<}{0.001}$ \\
\hline
\end{tabular}

Notes: Values are in the term of mean (Mean $\pm S D)$. Cl, confidence interval; ${ }^{\star} p<0.05$ relative to Group A.

The postpartum hemorrhage within $24 \mathrm{~h}$ did not show significant difference between the two groups (Table 4).

Table 4 Postpartum hemorrhage within $24 \mathrm{~h}$

\begin{tabular}{|lllll|}
\hline postpartum hemorrhage & Group $A(n=30)$ & Group B $(n=30)$ & Cl 95\% & P value \\
\hline $1 \mathrm{~h}$ & $42.83 \pm 19.72$ & $32.83 \pm 14.95^{\star}$ & $0.95-19.04$ & 0.03 \\
\hline $2 \mathrm{~h}$ & $33.5 \pm 23.7$ & $29.0 \pm 14.4$ & $-5.69-14.69$ & 0.38 \\
\hline $6 \mathrm{~h}$ & $32.8 \pm 24.69$ & $30.0 \pm 15.19$ & $-7.76-13.43$ & 0.59 \\
\hline $12 \mathrm{~h}$ & $30.9 \pm 27.88$ & $23.66 \pm 14.7$ & $-4.25-18.79$ & 0.21 \\
\hline $24 \mathrm{~h}$ & $19.0 \pm 12.7$ & $24.0 \pm 16.04$ & $-12.49-2.49$ & 0.19 \\
\hline
\end{tabular}

Notes: Values are in the term of mean (Mean $\pm S D$ ). $C l$, confidence interval; ${ }^{*} p<0.05$ relative to Group A.

The minimal respiration rates were not significantly different among groups, and no patient showed ventilatory depression. The sufentanil-related side effect like PONV, headache, dizziness, constipation and pruritus were not found among Groups. All parturients had the same Ramsey sedation score.

\section{Discussion}

Opioids, as a kind of analgesics, can effectively and potently relieve moderate-to-severe postoperative pain, and common opioids for PCIA are morphine, sufentanil and fentanyl [10-13]. Morphine, considered the most appropriate drug for PCIA, has enjoyed the widest application and the most study [10]. Nevertheless, its effect can be under the impact of its active metabolite, morphine-6-glucuronide, which can also generate respiratory depression, particularly for renal insufficiency patients as kidney mainly 
takes charge of metabolite excretion [14]. Sufentanil is a selective $\mu$-receptor agonist and usually serves for patient-controlled analgesia (PCA) relying on the quick peak, strong analgesic activity, as well as short half-life $[15,16]$. It does not have any active metabolite, just like fentanyl. Its potency is $5-10$ times than fentanyl, its parent drug $[17,18]$. Sufentanil has a lower respiratory depression incidence relative to the other opioids $[11,19]$. For sufentanil, the equilibration half-life is relatively rapid ( $t \frac{1}{2} k e 0 ; 6.2$ minutes) between plasma and brain, and that for morphine is 2.8 hours [20,21], and the action duration is a relatively shorter relative to morphine and fentanyl [22]. Sufentanil transfer into milk is not clear, while it has a similar safety profile to fentanyl. It has a low level in breast milk, making it impossible to bring into any obvious effects [23]. Considering above mentioned properties, sufentanil is regarded as a proper drug for PCIA. It enjoys the widest application for PCIA over China [12, 24], and also serves for controlling postoperative pain and relieving labor pain for surgery patients during anesthesia [25-27].

Study founds the dose of $2.5 \mu \mathrm{g} / \mathrm{kg}$ of sufentanil with background infusion as the most proper dose as it can better alleviate pain at activity and does not increase the side effects $48 \mathrm{~h}$ following surgery [28]. Combined with our work experience, the dose of sufentanil is set at $2 \mu \mathrm{g} / \mathrm{kg}$.

Apfel shows that the female gender, prior history of MS (motion sickness) or PONV, and nonsmoking were the main four risk factors of PONV [29], so we excluded these people. Although the panel of American pain society recommends that background infusion of opioids can enhance nausea and vomiting, our study shows that the PONV was not observed in both group. Regarding patients who use opioids after cesarean section, the reason for the extremely low incidence of PONV deserves further study.

Researchers have been attempted to establish scores for rating the severity regarding nausea, vomiting, and retching for patients receiving chemotherapy $[30,31]$. Nevertheless, the established score or index cannot well define PONV with clinical significance. Previously used techniques for measuring PONV include absolute counts or incidence rate, visual analogue scales (VAS), Likert scales, as well as treatment response. Wengritzky R proposes to use the VAS as screening tool for the rapid assessment in the clinical setting or as an audit tool[31], we choose it.

The uterine contraction pain refers to uterine contraction related intermittent cramping pain. The wound pain refers to the constant evoked pain over the wound and neighboring region. Postcesarean pain is different from most postoperative pain, and it includes a nociceptive barrage from incisional wound as well as the uterine contraction [32]. In our study, both types of pain were scored together. Because the pain of uterine contraction is enough to cover the wound pain, it was found in the study that many patients could not distinguish between the pain of the two, resulting in the same pain score. Our study found the higher VAS at 6 hours after surgery, which may lead to inability to effectively reduce the pain of uterine contraction by using sufentanil alone. Hsing-Wen Hsu found [32] that $20 \mathrm{mg}$ tenoxicam remarkably enhanced the ability of intravenous PCA morphine to relieve uterine cramping pain. Because it is not suggested to use NSAIDs for parturient in the postpartum period. Uterine contractions can reduce the amount of postpartum hemorrhage, and postpartum hemorrhage is one of the serious complications of cesarean section parturients, and massive hemorrhage is the direct cause of hysterectomy and 
maternal death [33]. our study found that Group B significantly reduces the pain of uterine contractions, but also reduces the amount of bleeding in parturients $1 \mathrm{~h}$ after surgery, directly reducing the adverse reactions of postpartum hemorrhage.

If the $\mathrm{l} / \mathrm{A}$ is lower, it means that the number of parturients attempts for each injection is higher, showing insufficient pain control. Besides, according to Sinatra et al [34], when the I/A ratio is lower, there may be a delay in obtaining the peak analgesic effect.

\section{Conclusion}

In comparison with the without background infusion, total consumption of sufentanil at 36 hours postoperative was much greater in the background infusion, but it can significantly reduce the uterine contraction pain and wound pain of 12 hours after cesarean section, significantly improve patient satisfaction, reduce the amount of bleeding after 1 hour, and does not increase the PONV incidence and respiratory depression in parturients.

\section{Material And Methods}

\section{Study design}

The study is a single center study. The experiments were performed in a random manner with two-arm parallel groups. The results are prospective. Methods were not changed following trial commencement.

\section{Ethical approval}

After Shanxi Bethune hospital's committee approval, consenting parturients were invited to participant in the prospective randomized study (NO. YXLL-SL-2020-017). We had obtained all subjects' written informed consent. This study was conducted in compliance with China Good Clinical Practice (GCP) and the tenets of the Declaration of Helsinki. Written informed consent was obtained from all parturients. We registered the study before patient enrollment at the Chinese Clinical Trial Register, a participant in the World Health Organization International Clinical Trials Registry Platform (registration number: ChiCTR2000037940, Principal investigator: Zhong-biao Nie, Registration date: 04/09/2020, URL: http://www.chictr.org.cn/edit.aspx? pid=60429\&htm=4).

\section{Patient population}

Inclusion criteria were: First delivery women in the Shanxi Bethune hospital, age between 18 and 40 years, cesarean section, BMI 18 to $28 \mathrm{~kg} / \mathrm{m}^{2}$, American Society of Anesthesiologists (ASA) grade I to II, no smoking history, opioid-naive adults.

The exclusion criteria were: parturients with severe heart, lung, liver, and kidney insufficiency, hypertension during pregnancy and preoperative fetal distress, as well as those with a history of mental or neurological 
diseases and opioid allergies; Opioid-tolerant parturients; the smoking history of; a history of motion sickness History; History of PONV.

Postoperative exclusion criteria: re-operation exploration, hemodynamic instability, which included serious arrhythmia, bleeding, as well as postoperative confusion. (Hemodynamic instability: $\mathrm{SBP}<90 \mathrm{mmhg}$ ).

\section{Anesthesia and PCA regimen}

All parturients received $500 \mathrm{~mL}$ of intravenous Lactated Ringer's solution before epidural analgesia. The needle-through-needle technique was adopted to conduct CSEA on all participants at L3-4 or L2-3 space. When patients showed hypotension (SBP $<90 \mathrm{mmHg}$ or the reduction $>20 \%$ of the basal SBP), they would receive $50 \mu \mathrm{g}$ of phenylephrine through intravenous injection, with fluid infusion accelerated. When SBP $>180 \mathrm{mmHg}$, they would receive $25 \mathrm{mg}$ of urapidil or $0.1 \mathrm{mg}$ of nicardipine through intravenous injection at a lowed speed. When HR<50 bpm, they would receive $0.2 \mathrm{mg}$ of atropine through intravenous injection. Following surgery, parturients would be sent to post-anesthesia care units (PACU). With stable vital signs and sensory block $\leq T 8$, parturients would be sent back to wards, connected with the PCIA pumps. PCIA pumps were stopped $36 \mathrm{~h}$ later.

According to body weight, $2 \mu \mathrm{g} / \mathrm{kg}$ sufentanil (Yichang Humanwell Pharmaceutical) was diluted to $100 \mathrm{ml}$ with normal saline. 60 Parturients had a PCIA analgesia protocol initiated consisting of a $0.5 \mathrm{~mL}$ bolus, and compared 2 Groups: no background infusion, 6 min lockout time (Group A). $2 \mathrm{~mL} / \mathrm{h}$ infusion, $10 \mathrm{~min}$ lockout time (Group B). A excel 'Random' function-generated randomization sheet was used to prepare sealed opaque envelopes. Qualified clinical research pharmacist who took charge of drug management kept the randomized sheet; prior to surgery, drugs were placed in a sealed opaque envelope, which could only be opened by a relevant anesthesiologist who took charge of managing anesthesia and PCIA setting following protocol in the operating room. Other investigators responsible for study endpoints assessment in PACU and ward after operation knew nothing about how groups were assigned. Parturients only labeled with the study number of patients could use all PCIA devices, therefore, both the parturients and medical care providers as well as investigators cannot recognize the PCIA regimen.

The parturients were instructed on the use of the PCIA pump and told them to press the button whenever they feel pain. Parturients were allowed to receive $100 \mathrm{mg}$ tramadol via intramuscular injection when they complained of pain $>7$ on VAS, and it was allowed to repeat the injection in necessity. The parturients were allowed to receive $10 \mathrm{mg}$ of intravenous metoclopramide as a rescue antiemetic as required. If patients still presented serious nausea persisted after being administered with two consecutive rescue antiemetics, PCIA would be interrupted for two hours and be restarted when serious nausea gradually vanished.

\section{Evaluation of outcomes}


The total amount of sufentanil consumed and the two pain categories was assessed, with scores of wound pain at rest (VAS-R) scores, and uterine cramping pain (VAS-U) in the first 36 postoperative hours being the major result measurement indexes. A $10-\mathrm{cm}$ visual analog scale (VAS) was used to record the pain intensity, in the range of 0 (no pain) to 10 (the worst pain imaginable). Secondary outcomes were the Ramsey sedation score (RSS) [35], assessed at the same time points, postpartum hemorrhage within 24 h. Injection/attempt (I/A) ratio. The I/A ratio was used to compare the number of the actual analgesic doses and that of the requested analgesic (attempts). In ideal conditions, the closer the I/A ratios get to 1 the better, which indicated that every patient injection should correspond to one attempt for injecting analgesic. A 4-point verbal scale was employed to rate the postoperative nausea and vomiting (PONV) (none, mild, moderate and severe respectively meaning no nausea, nausea but no vomiting, vomiting one attack and vomiting > one attack). All other side effects associated with sufentanil were dizziness, headache, constipation and pruritus. The situation of respiratory rate less than ten per min or oxygen saturation less than $90 \%$ for over $1 \mathrm{~min}$ is defined as respiratory depression [36]. A scale was adopted to measure the satisfaction of parturients with the PCIA for the past 36 hours, $(0=$ not satisfied, and $5=$ very satisfied). A trained nurse blinded to how the groups were assigned took charge of assessing the parturients. Heart rate and MAP were recorded postoperatively at $6,12,24$, and 36 hours.

\section{Statistics}

The primary endpoint pain level assessed by VAS was analyzed. The expected standard deviation of means was $6.9 \mathrm{~mm}$, and standard deviation of subjects was $20 \mathrm{~mm}$. The significance level was set at 0.05 and the power at 0.8 . Then sample size assumption was made through the software program PASS 11 (NCSS, LLC, Kaysville, Utah, USA) based on one-way ANOVA. And the calculated sample size was 30 in each group[28]. The SPSS software (version 20.0; IBM, USA) was applied to the statistical analyses. Measurement data are in the form of means \pm standard deviations $(M \pm S D)$ or the mean difference with $95 \%$ confidence interval [MD, 95\% Cl]. Categorical data are in the form of frequency and cases. Whether data were in line with a normal distribution was tested via the Kolmogorov-Smirnov test. Demographic and outcome data are summarized as $\mathrm{M} \pm \mathrm{SD}$. Measurement data in line with the normal distribution were compared, including VAS pain scores, $\mathrm{I} / \mathrm{A}$. The independent $\mathrm{t}$ test served for examining the total sufentanil consumption, satisfaction, postpartum hemorrhage. The $\chi^{2}$ test or Fisher's exact test served for examining categorical data including the incidence of side effects. $P$ values $<0.05$ exhibited statistical significance.

\section{Declarations}

\section{Consent}

The study had obtained all participants' written informed consent.

\section{Conflicts of Interest}

The authors declare no conflicts of interest regarding the paper. 


\section{Authors' Contributions}

All authors contributed substantially to this manuscript. Zhong-Biao Nie, and Ping Zhuang were involved in the design of the study. Ping Zhuang and Bin Lu were responsible for case collection. Ran Zhang, XianMei Cui, Tao Cao and Su-Xian Li were involved in collecting the data. Ran Zhang, Su-Xian Li helped in analyzing the data. Zhong-Biao Nie, and Ping Zhuang assisted in writing the manuscript. The final manuscript has been read and approved by all authors.

\section{Statement}

This study has been approved by Shanxi Bethune hospital's committee, consenting parturients were invited to participant in the prospective randomized study(NO. YXLL-SL-2020-017, date:06/05/2020). The Ethical review statement is attached below.

\section{References}

1. Kerai, S., K.N. Saxena and B. Taneja, Post-caesarean analgesia: What is new? Indian Journal of Anaesthesia, 2017. 61(3): p. 200.

2. Evans, K.C., et al., Effect of caesarean section on breast milk transfer to the normal term newborn over the first week of life. Arch Dis Child Fetal Neonatal Ed, 2003. 88(5): p. F380.

3. Analgesic efficacy and adverse effects of epidural morphine compared to parenteral opioids after elective caesarean section: A systematic review. European Journal of Pain, 2010. 14(9): p. 894.e1894.e9.

4. An audit of postoperative intravenous patient-controlled analgesia with morphine: Evolution over the last decade. European Journal of Pain, 2009. 13(5): p. 464-471.

5. Chou, R., et al., Management of Postoperative Pain: A Clinical Practice Guideline From the American Pain Society, the American Society of Regional Anesthesia and Pain Medicine, and the American Society of Anesthesiologists' Committee on Regional Anesthesia, Executive Commit. The Journal of Pain, 2016.

6. Etches, R.C., Respiratory depression associated with patient-controlled analgesia: a review of eight cases. Canadian Journal of Anaesthesia, 1994. 41(2): p. 125-132.

7. Lian, L.L., et al., Respiratory depression: An adverse outcome during patient controlled analgesia therapy. Journal of Clinical Anesthesia, 1996. 8(2): p. 151-156.

8. E, P. and Macintyre, Safety and efficacy of patient-controlled analgesia. British journal of anaesthesia, 2001.

9. Tayfun, et al., A background infusion of morphine enhances patient-controlled analgesia after cardiac surgery. Canadian Journal of Anesthesia, 2004.

10. Grass, J.A., Patient-Controlled Analgesia. Anesthesia and analgesia, 2005(No.5Suppl): p. S44-S61. 
11. Palmer, P.P. and R.D. Miller, Current and Developing Methods of Patient-Controlled Analgesia. Anesthesiology Clinics, 2010. 28(4): p. 587-599.

12. Zhang, Y., et al., To predict sufentanil requirement for postoperative pain control using a real-time method: A prospective observational cohort study. Medicine, 2016. 95(25): p. e3915.

13. Kim, K.M., Analysis of the current state of postoperative patient-controlled analgesia in Korea. 2016.

14. Osborne, R.J., S.P. Joel and M.L. Slevin, Morphine intoxication in renal failure: the role of morphine-6glucuronide..

15. Sedighinejad, A., et al., Magnesium Sulfate and Sufentanil for Patient-Controlled Analgesia in Orthopedic Surgery. Anesthesiology \& Pain Medicine, 2014. 4(1): p. e11334.

16. Savoia, G., M. Loreto and E. Gravino, Sufentanil: an overview of its use for acute pain management. Minerva Anestesiologica. 67(9 Suppl 1): p. 206.

17. Maciejewski, D., Sufentanil in anaesthesiology and intensive therapy. Anaesthesiology Intensive Therapy, 2013. 44(1): p. 35-41.

18. Kim, D.K., et al., Comparison of the Effects of Sufentanil and Fentanyl Intravenous Patient Controlled Analgesia after Lumbar Fusion. Journal of Korean Neurosurgical Society, 2017. 60(1): p. 54-59.

19. Bailey, P.L., et al., Differences in magnitude and duration of opioid-induced respiratory depression and analgesia with fentanyl and sufentanil. Anesthesia \& Analgesia, 1990. 19(7): p. 846-846.

20. Scott, J.C., J.E. Cooke and D.R. Stanski, Electroencephalographic quantitation of opioid effect: comparative pharmacodynamics of fentanyl and sufentanil. Anesthesiology, 1991. 74(1): p. 34-42.

21. L Tsch, J.R., et al., The Transfer Half-life of Morphine-6-glucuronide from Plasma to Effect Site Assessed by Pupil Size Measurement in Healthy Volunteers. Anesthesiology, 2001. 95(6): p. 13291338.

22. Hughes, M.A., P.S.A. Glass and J.R. Jacobs, Context-sensitive Half-time in Multicompartment. Anesthesiology, 1992. 76(3): p. 334-341.

23. Montgomery, A. and A.T.A.O. Hale, ABM clinical protocol \#15: analgesia and anesthesia for the breastfeeding mother. Breastfeeding Medicine the Official Journal of the Academy of Breastfeeding Medicine, 2017. 7(6): p. 547-53.

24. Nie, Y., et al., Effect of dexmedetomidine combined with sufentanil for post-caesarean section intravenous analgesia: a randomised, placebo-controlled study. European Journal of Anaesthesiology, 2014. 31(4): p. 197.

25. Sitsen, E., et al., A comparison of the efficacy of levobupivacaine $0.125 \%$, ropivacaine $0.125 \%$ and ropivacaine $0.2 \%$, all combined with sufentanil $0.5 \mathrm{microg} / \mathrm{mL}$, in patient-controlled epidural analgesia after hysterectomy under combined epidural and general anesthesia. Acta Anaesthesiologica Belgica, 2012. 63(4): p. 169.

26. Mendola, C., et al., Thoracic epidural analgesia in post-thoracotomy patients: comparison of three different concentrations of levobupivacaine and sufentanil. British Journal of Anaesthesia, 2009. 102(3): p. 418-423. 
27. Boselli, E., et al., Background Infusion Is Not Beneficial during Labor Patient-controlled Analgesia with $0.1 \%$ Ropivacaine plus $0.5 \mu \mathrm{g} / \mathrm{ml}$ Sufentanil. Anesthesiology, 2004. 100(4): p. 968-72.

28. Luming, et al., Dose determination of sufentanil for intravenous patient-controlled analgesia with background infusion in abdominal surgeries: A random study. Plos One, 2018.

29. Apfel, C.C.M.D., et al., A Simplified Risk Score for Predicting Postoperative Nausea and Vomiting: Conclusions from Cross-validations between Two Centers. Anesthesiology, 1999(No.3): p. 693.

30. Molassiotis, A., et al., Validation and Psychometric Assessment of a Short Clinical Scale to Measure Chemotherapy-Induced Nausea and Vomiting: The MASCC Antiemesis Tool. Journal of Pain \& Symptom Management, 2007. 34(2): p. 148-159.

31. Wengritzky, R., et al., Development and validation of a postoperative nausea and vomiting intensity scale. Br J Anaesth, 2010(2): p. 158-166.

32. Hsu, H.W., et al., Differential analgesic effect of tenoxicam on the wound pain and uterine cramping pain after cesarean section. Clinical Journal of Pain, 2003. 19(1): p. 55-58.

33. Alp, T., J.P. Souza and M. Gülmezoglu, New WHO recommendations on prevention and treatment of postpartum hemorrhage. International Journal of Gynaecology \& Obstetrics the Official Organ of the International Federation of Gynaecology \& Obstetrics, 2013. 123(3): p. 254-256.

34. Sinatra, R.S., et al., A comparison of morphine, meperidine, and oxymorphone as utilized in patientcontrolled analgesia following cesarean delivery. Anesthesiology, 1989. 70(4): p. 585.

35. Dawson, R., N.V. Fintel and S. Nairn, Sedation assessment using the Ramsay scale. Emerg Nurse, 2010. 18(3): p. 18-20.

36. Tsui, S.L., et al., An audit of the safety of an acute pain service. Anaesthesia, 1997(No.11): p. 10421047.

\section{Figures}




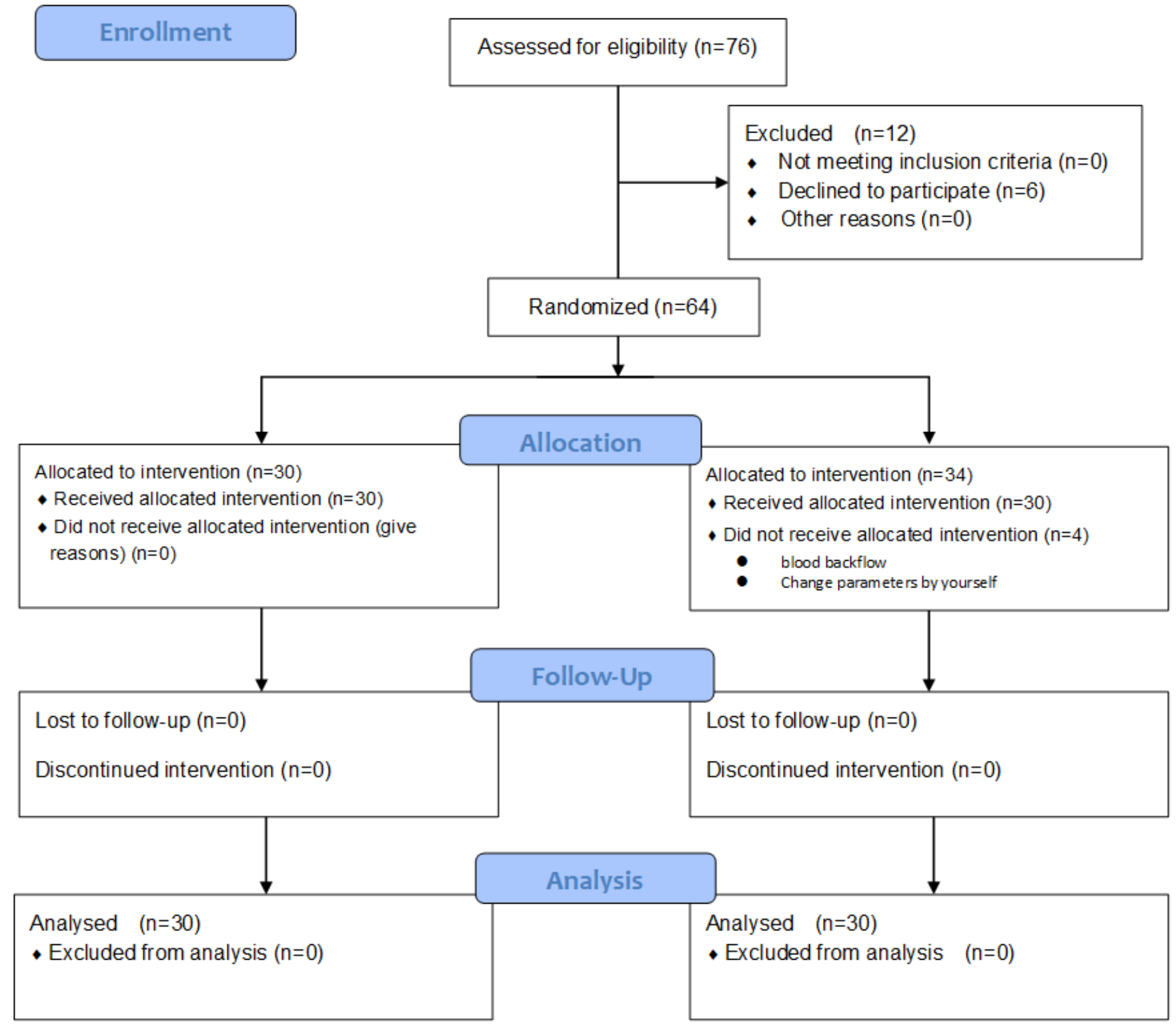

Figure 1

CONSORT recruitment flow diagram

\section{Supplementary Files}

This is a list of supplementary files associated with this preprint. Click to download.

- CONSORT2010Checklist.pdf 